\title{
Long non-coding RNA LINC00426 contributes to doxorubicin resistance by sponging miR-4319 in osteosarcoma
}

\author{
Lulin Wang ${ }^{1+}$, Yi Luo ${ }^{1 \dagger}$, Yiquan Zheng ${ }^{1}$, Lifeng Zheng ${ }^{2}$, Wenxiang Lin ${ }^{1}$, Zonglin Chen', Shichun Wu',
} Jinhong Chen ${ }^{1^{*}}$ and Yun Xie ${ }^{2^{*}}$

\begin{abstract}
Background: LINC00426 is a newly identified long non-coding RNA (IncRNA) with unacknowledged biological roles. Here we set out to characterize the expression status of LINC00426 in osteosarcoma and understand its mechanistic involvement in incidence of doxorubicin (Dox) resistance.

Methods: The relative expression of LINC00426 and miR-4319 was determined by real-time PCR. Cell viability and proliferation in response to LINC00426 silencing or miR-4319 over-expression was measured with CCK-8 kit and colony formation assay, respectively. The direct association between LINC00426 and miR-4319 was analyzed by pulldown assay with biotin-labelled probes.

Results: LINC00426 was significantly up-regulated in Dox-resistant osteosarcoma (OS) both in vitro and in vivo, which intimately associated with unfavorable prognosis. SiRNA-mediated knockdown of LINC00426 remarkably compromised cell viability and proliferation in Dox-resistant OS cells, which accompanied with decrease of IC50 and activation of caspase-3. We further predicted and validated the regulatory effects of miR-4319 on LINC00426 expression. Simultaneously, we provided evidences in support of direct binding between LINC00426 and miR-4319 by pulldown assay. Reciprocally negative regulation was observed between LINC00426 and miR-4319 each other. Conclusion: Ectopic introduction of miR-4319 significantly surmounted the Dox resistance in OS cells, while miR4319 inhibition in LINC00426-deficient cells greatly restore this phenotype. We uncovered the important contribution of LINC00426/miR-4319 to Dox resistance in osteosarcoma.
\end{abstract}

Reviewers: This article was reviewed by Bo Liang and Sinan Zhu.

Keywords: LINC00426, miR-4319, Osteosarcoma, Doxorubicin resistance

\footnotetext{
* Correspondence: Lulin6936@sina.com; Xyaku987654@126.com

${ }^{\dagger}$ Lulin Wang and Yi Luo contributed equally to this work.

'Department of Orthopaedics, Zhangzhou Affiliated Hospital of Fujian

Medical University, No.59, Shengli Road West, Xiang Cheng District,

Zhangzhou 363000, Fujian, China

${ }^{2}$ Department of Orthopaedic Trauma, Trauma Center of Fujian, The First Affiliated Hospital of Fujian Medical University, No.20, Chazhong Road,

Taijiang District, Fuzhou 350005, Fujian, China
}

C C The Author(s). 2020 Open Access This article is licensed under a Creative Commons Attribution 4.0 International License, which permits use, sharing, adaptation, distribution and reproduction in any medium or format, as long as you give appropriate credit to the original author(s) and the source, provide a link to the Creative Commons licence, and indicate if changes were made. The images or other third party material in this article are included in the article's Creative Commons licence, unless indicated otherwise in a credit line to the material. If material is not included in the article's Creative Commons licence and your intended use is not permitted by statutory regulation or exceeds the permitted use, you will need to obtain permission directly from the copyright holder. To view a copy of this licence, visit http://creativecommons.org/licenses/by/4.0/. The Creative Commons Public Domain Dedication waiver (http://creativecommons.org/publicdomain/zero/1.0/) applies to the data made available in this article, unless otherwise stated in a credit line to the data. 


\section{Reviewers' comments}

Reviewers' comments: (reviewer 1, Bo Liang)

This is a solid study with convincing results. My comments are amended below. 1. The writing of this paper should be improved. For example, the background of the abstract should include necessary information about LINC00426 and the aim of the current study. 2. More details are required to establish Dox resistance cell lines. This is essential information. 3. The figures are too crowded with many texts in the figure. Please refine. 4. The list of abbreviations is not complete. 5. The significance of this study should be expanded in the conclusion section.

Response:

1. The writing of this paper should be improved. For example, the background of the abstract should include necessary information about LINC00426 and the aim of the current study.

Reply:

We appreciate the reviewer's kind suggestion and therefore we provided the background into LINC00426 and aim of our study in abstract section in page 3 line 2-3.

2. More details are required to establish Dox resistance cell lines. This is essential information.

Reply:

Thanks for your kind reminder, we have made an addition in the revised manuscript.

3. The figures are too crowded with many texts in the figure. Please refine.

Reply:

Thanks for your constructive suggestions, we have refined the figures in the revised version.

4. The list of abbreviations is not complete

Reply:

We apologize for this negligence and all abbreviations were listed in page 17 line 9-13 now.

5. The significance of this study should be expanded in the conclusion section

Reply:

We appreciated the reviewer's invaluable suggestion and the significance of our study has been greatly highlighted in the conclusion section in page 17 line 36.

Reviewers' comment: (reviewer 2, Sinan Zhu).

1. How was LINC00426 chosen in study from all other long non-coding RNAs? 2. What about the microRNAs could interact with this LINC00426? 3. Several statistical methods have been employed in the study. Method should be specified in the figure legends.

Response:

1. How was LINC00426 chosen in study from all other long non-coding RNAs?

Reply:
I really appreciate your concern about that. LINC00426 has been concerned in an occasional circumstance when we made the investigation of osteogenesis (a main research interests of our group), we ought to explore the functions and underlying mechanisms of LINC00426 in osteosarcoma. Previous research reported elevated LINC00426 was associated with osteosarcoma tumorigenesis and pulmonary metastasis [1], which indicated LINC00426 may play an oncogenic role in osteosarcoma, so we chose LINC00426 for further study.

[1] L. Xie, Z.H. Yao, Y. Zhang, D.Q. Li, F.D. Hu, Y.D. Liao, L. Zhou, Y.H. Zhou, Z.Y. Huang, Z.W. He, L. Han, Y.H. Yang, Z.Z. Yang, Deep RNA sequencing reveals the dynamic regulation of miRNA, lncRNAs, and mRNAs in osteosarcoma tumorigenesis and pulmonary metastasis, Cell Death Dis, 9 (2018).

2. What about the microRNAs could interact with this LINC00426?

\section{Reply:}

I really appreciate your concern about that. In Fig. 3, we first predicted the miRNA candidates by miRcode (http:// www.mircode.org/mircode) and miR-4319 was chosen as the novel function-related miRNA for further study.

Luciferase reporter assay showed that miR-4319 could interact with LINC00426 (Fig. 3b, c); biotin-RNA pulldown assay indicated that miR-4319 could bind to LINC00426 directly (Fig. 3d, e, f); besides, LINC00426 acted as a "sponge" to negatively regulate the expression of miR-4319 in OS cells (Fig. 3g, h).

3. Several statistical methods have been employed in the study. Method should be specified in the figure legends.

Reply:

Thanks for your kind reminder, we have made an addition in the revised manuscript.

\section{Background}

Osteosarcoma (OS) originates from the mesenchymal cells and is the most common primary bone malignancies affected children and adolescent, which is estimated to account for around $5 \%$ of all pediatric tumors [1]. The 5-year overall survival of OS based on the follow-up survey results is significantly improved in the last decade, which is greatly attributed to the advances in both of surgery and multiple combinational chemotherapy [2]. Acknowledged risk factors to this disease include bone dysplasia, TP53 mutant Li-Fraumeni syndrome and Rothmund-Thomson syndrome and frequent familiar genetic aberrance in chromosome 13q14 deletion, which consequently inactivates the retinoblastoma gene and links to high risk of osteosarcoma development [3]. Regular diagnosis of OS depends on X-ray and confirmative CT, PET, bone scan, MRI and surgical biopsy. The complete radical surgical resection is the treatment 
of choice in OS, which is applicable to as much as $90 \%$ of patients and clinical outcomes may be partially compromised by some complications including infection, prosthetic loosening and non-union, or local tumor recurrence. In this regard, current standard therapy of OS is combination of limb-salvage orthopedic surgery and high-dose of chemotherapeutic drugs such as methotrexate and leucovorin. Despite of the success of chemotherapy for osteosarcoma, the intrinsic and acquired drug resistances tremendously limited its clinical benefits [4]. The mechanistic elucidation of molecular events underlying the incidence and development of drug resistance will definitely guide our efforts toward more efficient and specific regimen for clinical management.

Long non-coding RNA (lncRNA) is defined as class of oligonucleotide longer than $200 \mathrm{nt}$ without protein-coding potentials [5]. Accumulative evidences suggest the involvement of lncRNAs in multiple biological processing including cell differentiation, proliferation, apoptosis, metastasis, drug resistance and stemness. LINC00426 is a newly identified lncRNA with unacknowledged biological roles [6], which make it pretty novel to systematically address its function in the initiation and progression of osteosarcoma. The conventional view implicated that lncRNAs involve in gene transcription regulation via complexation with multiple factors including activator, suppressor, initiation protein, elongation protein and termination proteins in either gene-specific or -nonspecific ways [7]. In the recent decades, the reciprocal regulation between lncRNAs and microRNAs (miRs) is increasingly recognized, which antagonistically and consequently contribute to the fine-tune of target gene expression in distinct cell contexts. The sponge lncRNA concept is proposed to explain this phenomenon, and numerous sponging lncRNAs are identified so far participating in diverse physiological and pathological processing [8]. Here we focused on LINC00426 and its mechanistic involvements in osteosarcoma at both in vitro and in vivo levels. We further pursue the understanding into the molecular mechanism along the competing endogenous RNA direction. Our data will unmask the mysterious roles of LINC00426 in osteosarcoma, at least under the condition of Dox resistance.

miRs are increasingly documented to be critically involved in the tumor biology. Among which, the tumor suppressor functions of miR-4319 have been uncovered in array of human malignancies previously [9-11]. Here our data addressed an unrecognized role of miR-4319 in drug resistance in osteosarcoma, which might hold potential promises for therapeutic exploitation.

\section{Materials and methods}

\section{Cell culture}

Human OS cell lines (MG63, KHOS and U2OS) were obtained from the American Type Culture Collection
(ATCC, NY, USA) and cultured in DMEM medium containing 15\% fetal bovine serum (FBS, Gibco, MA, USA) and $1 \%$ penicillin/streptomycin (Invitrogen, MA, USA) at $37^{\circ} \mathrm{C}$ in humidified $\mathrm{CO}_{2}(5 \%)$ incubator. The Dox resistance cell lines (MG63/DXR, KHOS/DXR) were established by serially increased concentration of Dox as described previously [12]. Briefly. the OS cells were continuously cultured in complete medium supplemented with $0.0035 \mu \mathrm{M}$ Dox. The second (R2) generation was developed by continuous exposure of the corresponding R1 cells to $0.035 \mu \mathrm{M}$ Dox. The third (R3) generation of Dox-resistant OS cells was generated by culturing the OS R2 cells in the continuous presence of $0.35 \mu \mathrm{M}$ Dox. A cell line was considered as stable when the growth rate of the treated cells to a given Dox concentration was constant for at least 30 days.

\section{Cell transfection}

Indicated cells were seeded into 6-well plate the day before transfection. Transfection was performed with Lipofectamine 3000 (Invitrogen, MA, USA) according to the manufacturer's recommendation. The oligo sequences used in this study were provided as below:

si-LINC00426-1: 5'-GGCGCTATTTCGGCTCATTAT-3';

si-LINC00426-2: 5' -GCGCTATTTCGGCTCATTATA-3';

si-NC: 5' -TTCTCCGAACGTGTCACGT-3'; miR-4319: 5'-UGCUCCCUGAGGACGUUAUAUGA-3'; miR-NC: 5' -UGUGCAAAUCUAUGCAAAACUGA-3'.

\section{Real-time PCR}

Total RNA was extracted from either cells or tissues with TriZol reagent (Invitrogen, MA, USA). The singlestrand cDNA was prepared using the PrimeScript RT Reagent Kit (Takara, Dalian, China). The relative expression of LINC00426 and miR-4319 was determined with SYBR Green MasterMix Kit (Roche, Basel, Switzerland) on ABI PRISM 7900 HT System (Applied Biosystems, CA, USA). GAPDH and U6 were employed as reference control. All primer sequences were listed as follows:

miR-4319 RT prime: 5'-GTCGTATCCAGTGCAGGG TCCGAGGTATTCGCACTGGATACGACGTGGCT-3';

U6 RT prime: 5'-GTCGTATCCAGTGCAGGGTCCG AGGTATTCGCACTGGATACGACAAAATATGGAA-3';

LINC00426 qRT-PCR Forward: 5'-CAAGAAGACAGG GACAAGC-3';

LINC00426 qRT-PCR Reverse: 5'-ACTGAGTACC CAGCCAAAG-3';

GAPDH qRT-PCR Forward: 5'-CATGAGAAGTATGA CAACAGCCT-3';

GAPDH qRT-PCR Reverse: 5'-AGTCCTTCCACGAT ACCAAAGT-3'; 
miR-4319 qRT-PCR Forward: 5'-CACCCAGAGC

AAAGCCAC-3';

miR-4319 qRT-PCR Reverse: 5'-GTGCAGGGTC

CGAGGT-3';

U6 qRT-PCR Forward: 5'-TGCGGGTGCTCGCT

TCGGCAGC-3';

U6 qRT-PCR Reverse: 5' -GTGCAGGGTCCGAGGT-3' .

\section{Cell counting kit (CCK)-8 assay}

CCK-8 kit (Dojindo, Kumamoto, Japan) was used to measure cell viability. Briefly, 4000 indicated cells/well were seeded into 96-well plates in triplicated and subjected to specified transfection for $48 \mathrm{~h}$. CCK- 8 working solution was added and allowed for reaction for $2 \mathrm{~h}$ at $37^{\circ} \mathrm{C}$. Absorption at $450 \mathrm{~nm}$ was determined with microplate reader (Biotek, VT, USA) and relative cell viability was calculated accordingly.

\section{Colony formation assay}

Cell proliferation was evaluated by colony formation assay. The transfected cells (1000 cells) were prepared into 6-well plate in triplicate and continuously cultured for 2 weeks. The former colonies were fixed with PFA (4\% in PBS) first and stained with crystal violet $(0.05 \%$ in PBS) for $10 \mathrm{~min}$. Colony number was counted under light microscope and representative images were captured and presented.

\section{Caspase-3 activity assay}

The indicated cells were collected by trypsin digestion and caspase- 3 activity was determined with Cells were treated with different concentrations of MTX, ADM or DDP for $72 \mathrm{~h}$ and then collected with trypsin. The caspase-3 activity was measured with Caspase-3 Activity Assay Kit (Merck, MO, USA) according to the manufacturer's manual. Briefly, cell lysate was prepared in the supplied lysis buffer and substrate was added for $2 \mathrm{~h}$-incubation at $37^{\circ} \mathrm{C}$ in the dark. The absorption at $405 \mathrm{~nm}$ was recorded with microplate reader and relative caspase- 3 activity was calculated accordingly.

\section{Luciferase reporter assay}

LINC00426-driven luciferase reporter plasmids were constructed by PCR-Restriction Digestion-Ligation method. The mutant was generated by mutagenesis PCR method. The primer sequences used for PCR were provided as below. Both luciferase plasmids and miR-4319 were cotransfected into MG63/DXR and KHOS/DXR cells in triplicate in 6 -well plate $\left(1 \diamond 10^{6}\right.$ cells/well). The cell lysates were collected and dual luciferase activities were determined with Dual-Luciferase Reporter Assay System (Promega, WI, USA).

LINC00426 PCR Forward: 5'-AATTCTCGAG ACTCGGCCATGAAAGTC-3';
LINC00426 PCR Reverse: 5'-AATTGCGGCCGC TGTCTTCCAGTAAGACTTTA-3';

LINC00426 mutagenesis PCR Forward: 5'-CACCAC TTGTCTCAGAGGAA-3';

LINC00426 mutagenesis PCR Reverse: 5'-TTCCTC TGAGACAAGTGGTG-3' .

\section{Pull-down assay}

RNA was completely removed by RNase A incubation. Biotin-labeled probes were incubated with cell lysates from indicated cells $\left(2.5 \times 10^{7}\right)$ for one hour at room temperature. Pulldown assay was performed with streptavidin-coated agarose beads (Invitrogen, MA, USA) and enriched RNA specimen was reversely transcribed into cDNA as described previously. The relative abundance of target transcript was determined by realtime PCR. The probe sequences used in this study were provided as below:

Sense DNA probe: 5'-Biotin-GTCAGGACACAGCA AATGGGGGATCT-3';

Antisense DNA probe: 5' - Biotin-AGATCCCCCA TTTGCTGTGTCCTGAC-3';

Bio-miR-NC: 5 '-Biotin-UGUGCAAAUCUAUGCAAA ACUGA-3';

Bio-miR-4319: 5' -Biotin-UGCUCCCUGAGGACGUUA UAUGA-3 .

\section{Statistical analysis}

Results were presented as the mean values \pm SD unless specified and analyzed with two-tailed $t$-test, one- or two-way ANOVA with a Bonferroni post hoc test. A value of $p<0.05$ was considered significantly different.

\section{Results}

Expression level of LINC00426 in OS closely correlated with doxorubicin resistance and clinical outcomes

We first determined the relative expression of LINC00426 in three established doxorubicin-resistant OS cell lines in comparison with the sensitive parental cells. As shown in Fig. 1a, dramatic increases of LINC00426 transcript were observed in all of three cell lines, which indicated the intimate linkage between high abundance of LINC00426 with doxorubicin resistance at least in vitro. The up-regulation of LINC00426 in OS patients was further confirmed in 50 paired OS clinical samples (Fig. 1b). Notably, the significant higher level of LINC00426 was noticed in doxorubicin-sensitive patients compared with the resistant counterparts (Fig. 1c). Consistently, the Kaplan-Meier analysis demonstrated that low expression of LINC00426 significantly associated with better clinical outcomes (Fig. 1d). Therefore, both in vitro and in vivo data suggested that LINC00426 closely correlated with doxorubicin resistance and clinical survival. 


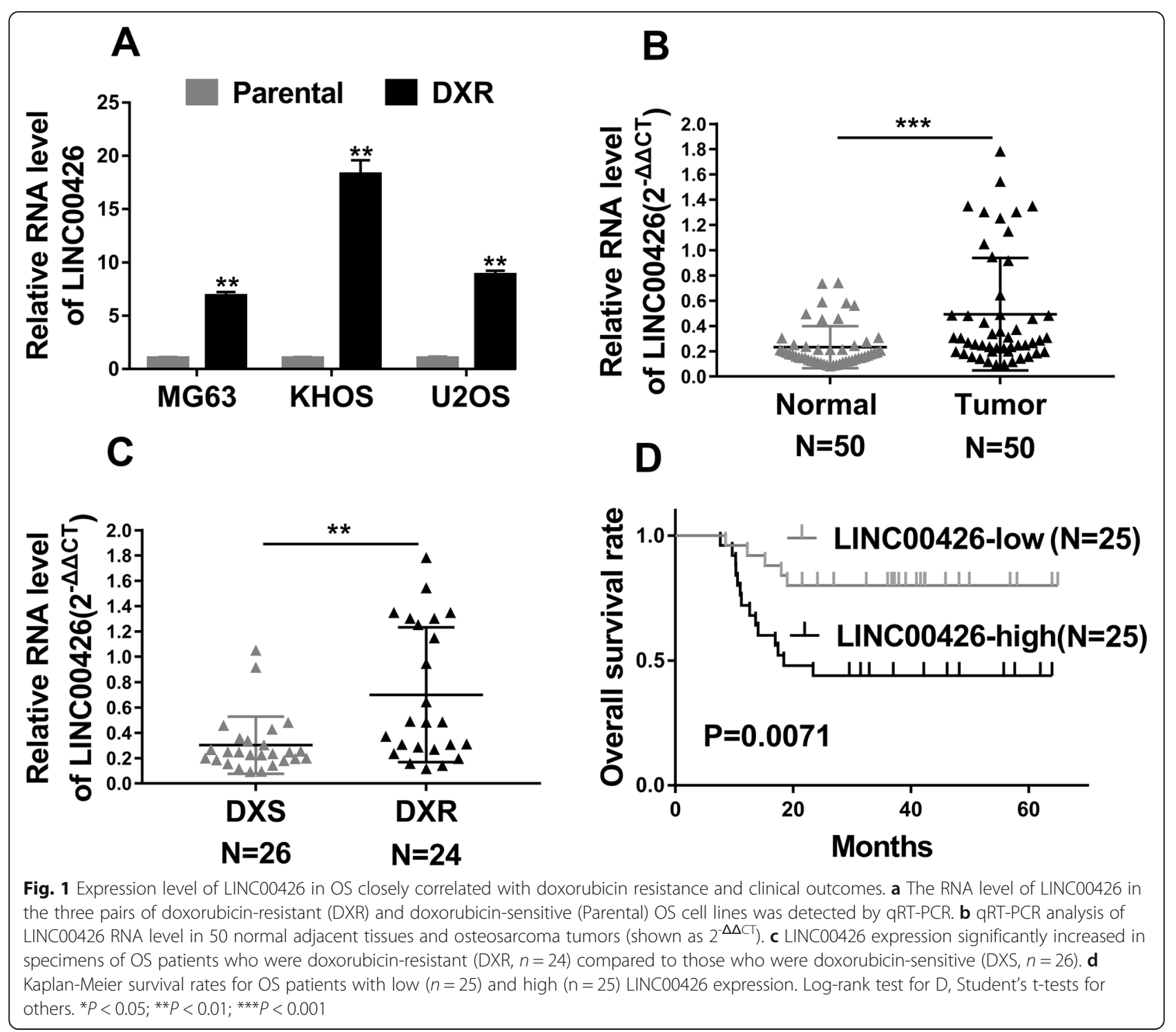

LINC00426 inhibition re-sensitized OS cells to doxorubicin Our previous results indicated the causal relation between high abundance of LINC00426 with doxorubicin resistance and clinical outcomes, which prompted us to investigate the potential benefits of LINC00426 inhibition in this scenario. Two independent siRNAs were employed to specifically silenced LINC00426 in both MG63/DXR and KHOS/DXR cells, and the knockdown efficiency was experimentally confirmed by real-time PCR (Fig. 2a). Cell viability measured with CCK-8 kit unambiguously was greatly compromised by LINC00426 depletion in both cells (Fig. 2b, c). Furthermore, the cell proliferation was significantly suppressed by LINC00426-specific siRNAs in both cell lines as well in comparison with scrambled negative control (Fig. 2d). Most importantly, the relative sensitivity to doxorubicin was markedly restored upon LINC00426 knockdown as indicated by the decrease of
IC50 value (Fig. 2e), which was accompanied by the remarkable increase of caspase-3 activity in LINC00426-deficient cells (Fig. 2f). Therefore, our data clearly demonstrated that LINC00426-silencing fundamentally contributed to the re-sensitization of resistant cells to doxorubicin treatments.

LINC00426 acted as a "sponge" of miR-4319 in OS cells To better understand the mechanistic involvements of LINC00426 in osteosarcoma, we next employed the bioinformatic tool miRcode (http://www.mircode.org/mircode) to predict and discover the possible target microRNA of LINC00426. Number of microRNAs were predicted with affinity to LINC00426 and miR-4319 was the top one in the list. The alignment between miR-4319 and either wild-type or mutant LINC00426 sequences were illustrated in Fig. 3a. To interrogate the potentially 


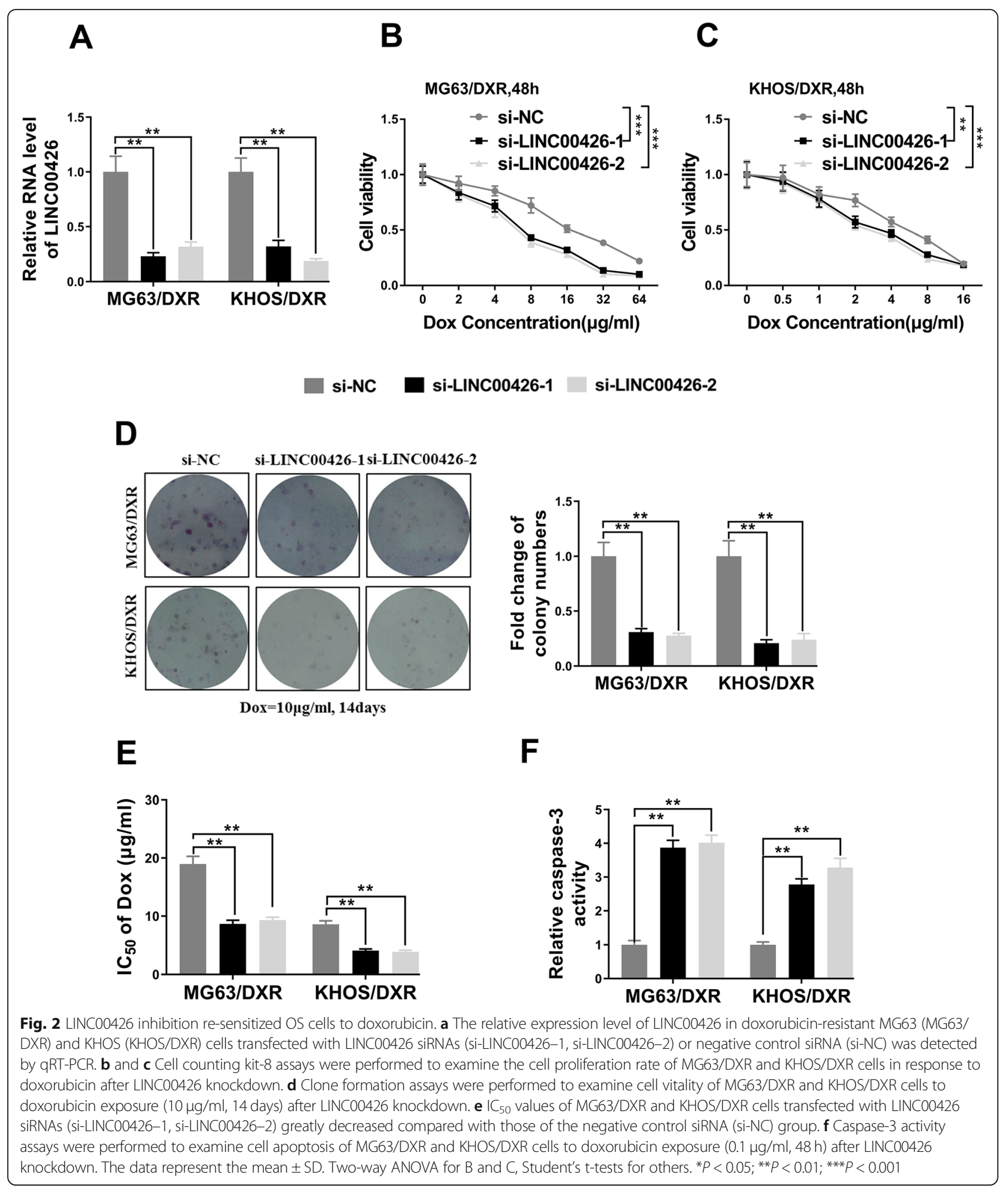

regulatory effects of miR-4319 on LINC00426, we constructed LINC00426-fused luciferase reporter plasmids. Co-transfection with miR-4319 greatly suppressed the LINC00426-driven luciferase activity in MG63/DXR cells in comparison with negative control, which was readily abolished by the mutation introduced into the putative recognizing site in LINC00426 transcript (Fig. 3b). Similar observation was confirmed in KHOS/DXR cells as shown in Fig. 3c. The binding between LINC00426 and miR-4319 was directly analyzed by pulldown assay with 


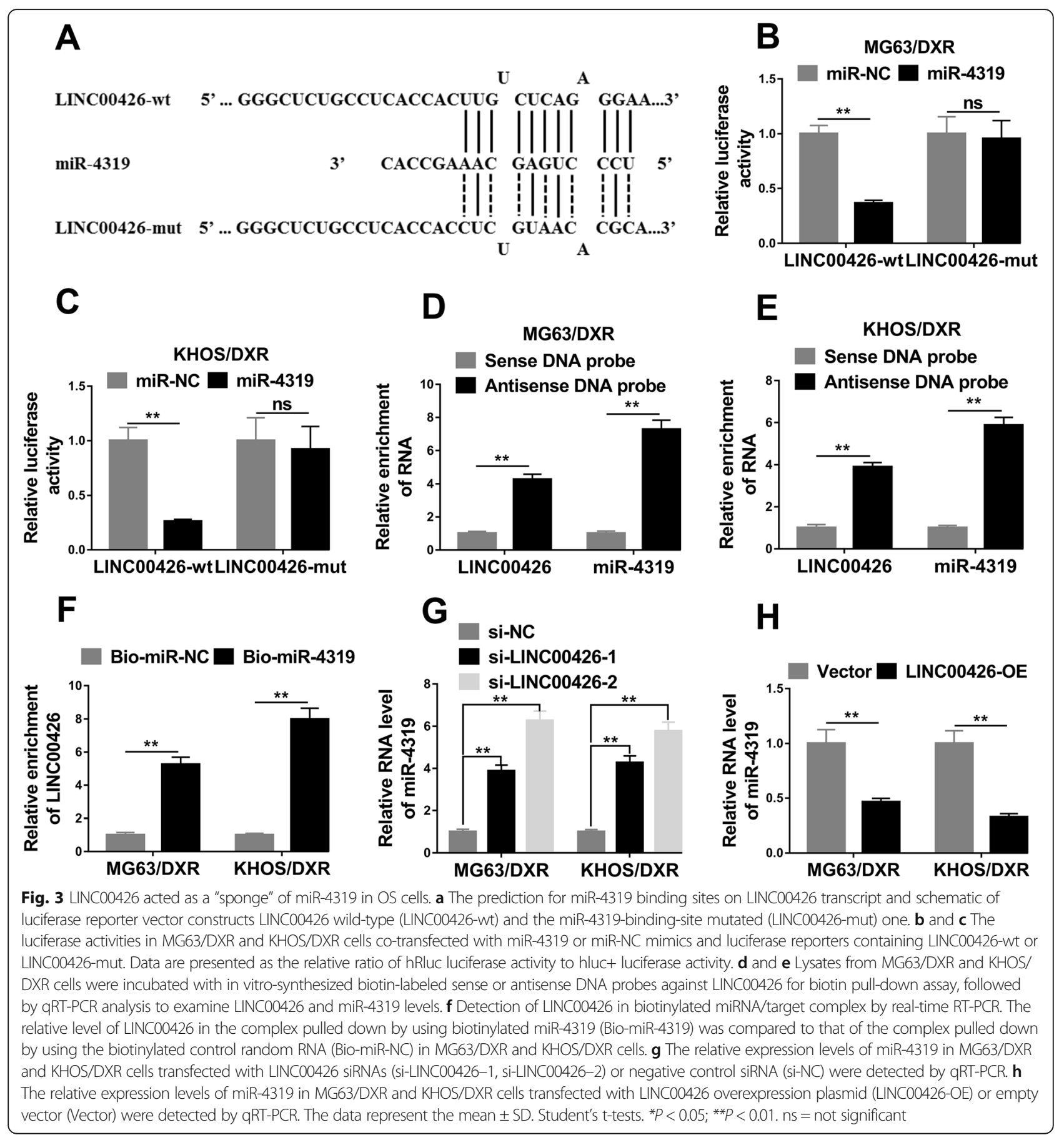

biotin-labelled probes. As shown in Fig. 3d and e, remarkable enrichments of both LINC00426 and miR-4319 were observed with antisense DNA probe other than sense DNA probe in both cell lines. Reciprocally, LINC00426 transcripts were significantly enriched in the miR-4319 pulldown complex in comparison with biotin-labelled negative control (Fig. 3f). We further noticed that endogenous miR4319 was greatly up-regulated in LINC00426-deficient cells and inhibited by exogenously introduced LINC00426 expression (Fig. 3g, h). Taken together, we provided evidences in support of the sponging function of LINC00426 against miR-4319 in osteosarcoma.

MiR-4319 acted as an inhibitor in the OS doxorubicin resistance

Our previous data suggested the negative regulation of LINC00426 on miR4319 expression in OS, which might functionally mediate the pathological roles of LINC00426 
in this disease. To clarify this possibility, we forced overexpression of miR-4319 in MG63/DXR and KHOS/DXR cells (Fig. 4a). The ectopic expression of miR-4319 significantly inhibited cell viability as indicated by the CCK- 8 results obtained from both cell lines (Fig. 4b, c). Consistently, cell proliferation was compromised as well by miR-4319 overexpression (Fig. $4 \mathrm{~d}$ ). The sensitivity to doxorubicin was also greatly improved by miR-4319 overexpression as implicated by the decrease in IC50 value of Dox (Fig. 4e). Again, the relative caspase-3 activity was remarkably restored by ectopic introduction of miR-4319 (Fig. 4f). In summary, our data suggested that miR-4319 acted as an inhibitor against doxorubicin resistance occurrence in OS.

\section{LINC00426 promoted OS doxorubicin resistance by sponging miR-4319}

Although we demonstrated the sponging function of LINC00426 against miR-4319 and improving effects of miR-4319 on Dox resistance in OS cells, whether miR-

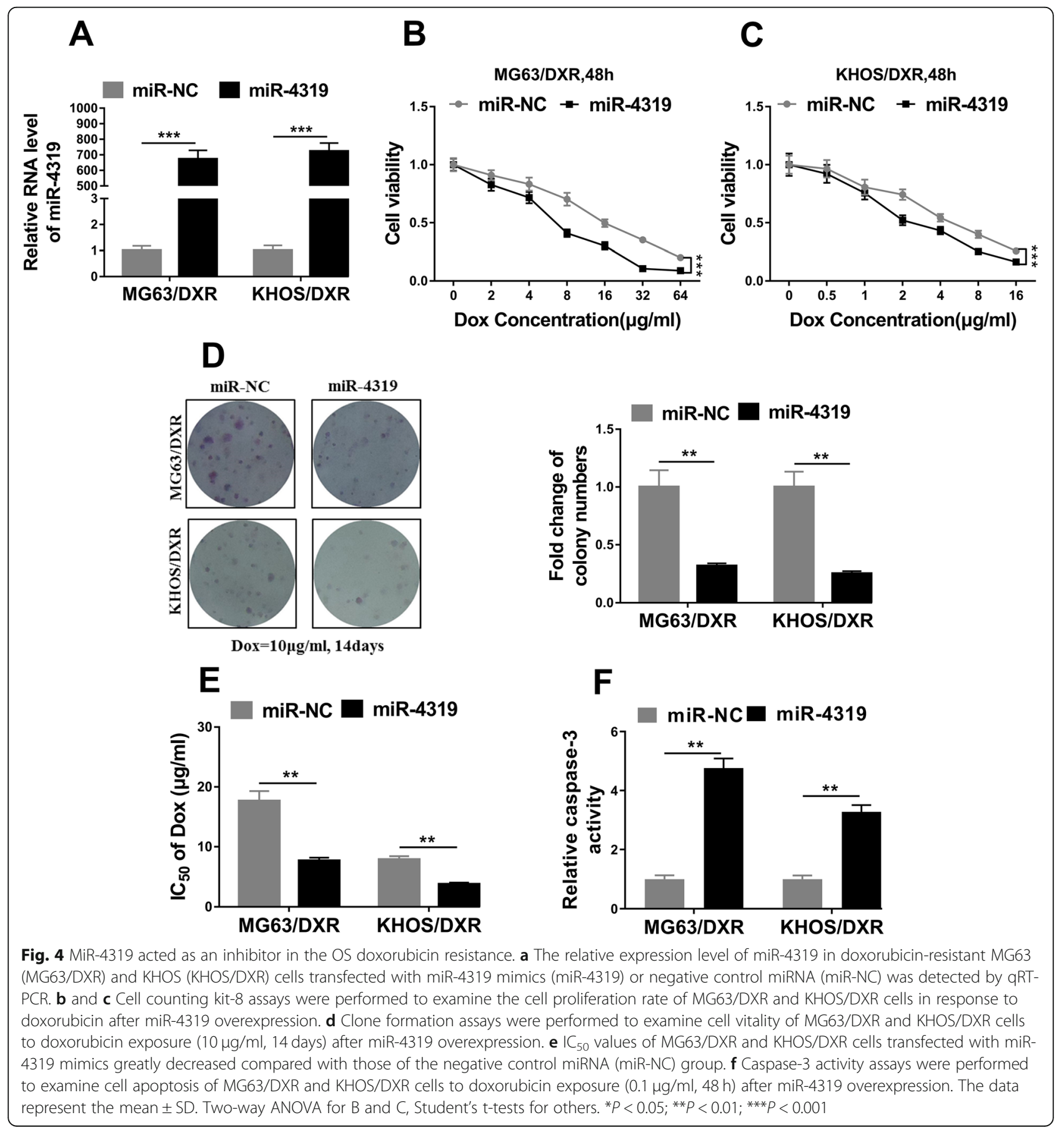


4319 predominantly contributed to doxorubicin resistance downstream LINC00426 was still to be addressed. To this end, we specifically inhibited miR-4319 in the LINC00426-deficient cells, the relative expression of miR-4319 in this scenario was validated by real-time PCR (Fig. 5a). The compromised cell viability elicited by LINC00426 deficiency was almost completely restored by miR-4319-inhibitor in both MG63/DXR and KHOS/ DXR cells (Fig. 5b, c). Similarly, the cell proliferation inhibited by si-LINC00426 was stimulated by cotransfection with miR-4319-inhibitor (Fig. 5d). Further analysis demonstrated that miR-4319 inhibition in the
LINC00426-silenced cells remarkably increased IC50 value of Dox, which suggested the re-occurrence of drug resistance under this condition (Fig. 5e). Consistently, the activation of caspase-3 was dramatically suppressed by miR-4319-inhibitor in both cell lines (Fig. 5f). Taken together, our data indicated that LINC00426 promoted Dox resistance in OS via sponging miR-4319.

\section{Discussion}

The critical contributions of lncRNAs in human malignancies were increasingly acknowledged [13]. In this study, we concentrated on the mechanistic involvements

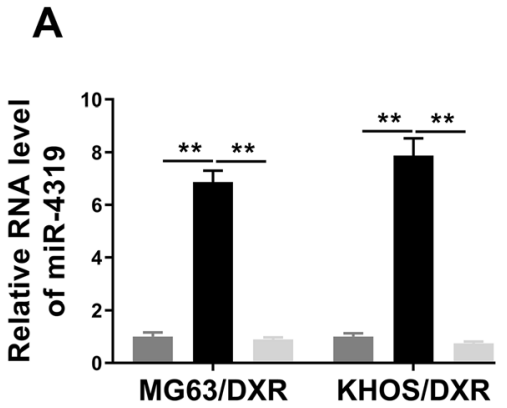

\section{B MG63/DXR,48h}

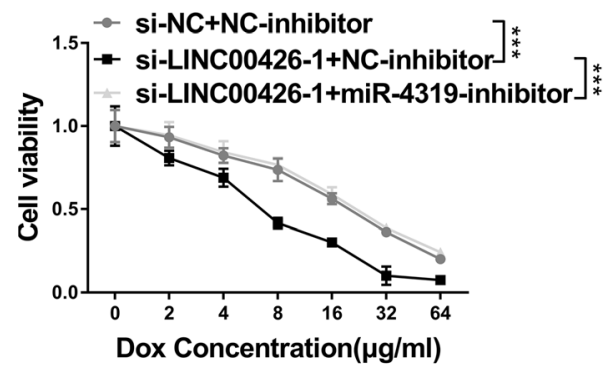

si-NC+NC-inhibitor

si-LINC00426-1+NC-inhibitor

si-LINC00426-1+miR-4319-inhibitor

C

D

KHOS/DXR,48h
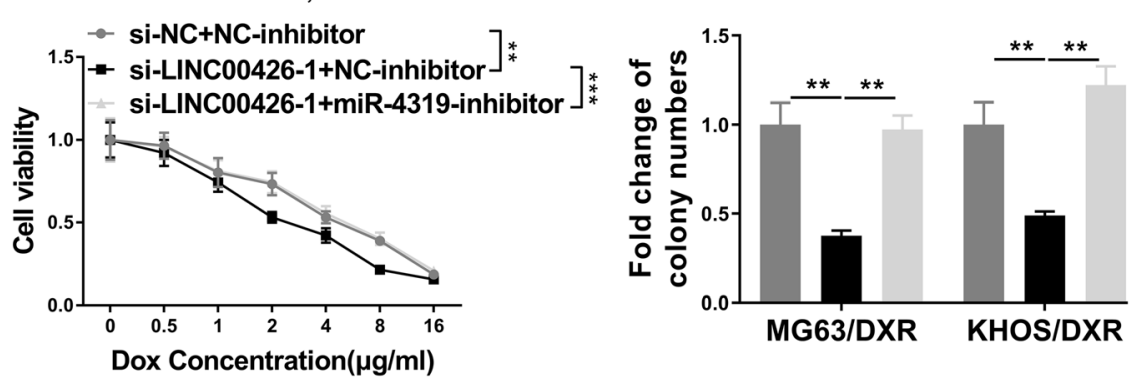

E

$\mathbf{F}$
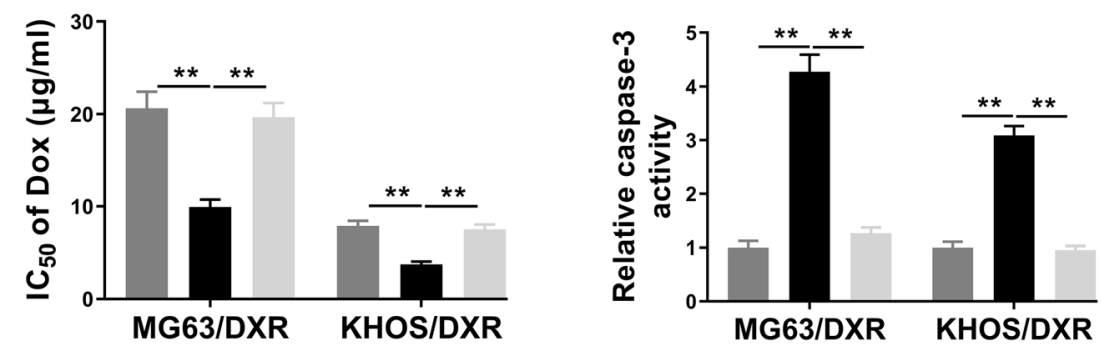

Fig. $\mathbf{5}$ LINC00426 promoted OS doxorubicin resistance by sponging miR-4319. a-f MG63/DXR and KHOS/DXR cells were co-transfected with siNC + NC-inhibitor, si-LINC00426-1 + NC-inhibitor or si-LINC00426-1 + miR-4319-inhibitor. a The RNA levels of miR-4319 in these co-transfected MG63/DXR and KHOS/DXR cells were determined by qRT-PCR. $\mathbf{b}$ and $\mathbf{c}$ Cell counting kit-8 assays were performed to examine the cell proliferation rate of these co-transfected MG63/DXR and KHOS/DXR cells in response to doxorubicin. $\mathbf{d}$ Clone formation assays were performed to examine cell vitality of these co-transfected MG63/DXR and KHOS/DXR cells to doxorubicin exposure ( $10 \mu \mathrm{g} / \mathrm{ml}, 14$ days). e $I C_{50}$ values of these co-transfected MG63/DXR and KHOS/DXR cells. $\mathbf{f}$ Caspase-3 activity assays were performed to examine cell apoptosis of these co-transfected MG63/DXR and KHOS/DXR cells to doxorubicin exposure $(0.1 \mu \mathrm{g} / \mathrm{ml}, 48 \mathrm{~h})$. The data represent the mean \pm SD. Two-way ANOVA for B and C, Student's t-tests for others. ${ }^{*} P<0.05 ;{ }^{* *} P<0.01 ;{ }^{* * *} P<0.001$ 
of LINC00426 in osteosarcoma. Our results first uncovered the aberrant over-expression of LINC00426 in doxorubicin-resistant OS cell lines in comparison with parental counterparts. In vivo analysis manifested the general up-regulation of LINC00426 in OS tumor and specifically elevated in the Dox un-responding patients. Higher LINC00426 abundance intimately associated with unfavorable prognosis as well, which might be due to the poorer response to Dox treatment. Employment of LINC00426-specific siRNAs in Dox-resistant cells significantly compromised both cell viability and proliferation, which was accompanied with improvement in response to Dox treatment and restoration of caspase-3 activation. With aid of bioinformatics tool, we predicted and experimentally validated that miR-4319 as direct target of LINC00426. Exogenous miR-4319 greatly suppressed the LINC00426-driven luciferase activities, and this inhibitory effect was readily abolished by the mutation introduced into LINC00426 transcript. The direct binding between LINC00426 and miR-4319 was experimentally validated with pulldown assay, wherein significant enrichment of both LINC00426 and miR-4319 was observed in biotinlabelled anti-sense DNA probe and vice versa. The reciprocal regulation between LINC00426 and miR-4319 was also confirmed in the Dox resistant cell lines. More importantly, ectopic supplementation with miR-4319 greatly inhibited cell viability and proliferation, which was accompanied with decrease of IC50 value of Dox and activation of caspase-3. Oppositely, inhibition of miR-4319 remarkably restored both cell viability and proliferation in LINC00426-deficient cells. Simultaneously, co-transfection with miR-4319inhibitor led to re-occurrence of Dox resistance as indicated by the increase of IC50 value and decrease of caspase- 3 activation. In summary, our data elucidated that high abundance of LINC00426, via negative regulation of miR-4319 expression, consequently contributed to the Dox resistance incidence in OS.

Drug resistance was inevitable for cancer chemotherapy and number mechanisms underlying this phenomenon were uncovered so far [14]. The fundamental contribution of lncRNAs in this scenario has increasingly received attentions from research community. For instance, Bai et al. showed that lncRNA LOXL1-AS1/miR-let-7a-5p/EGFRrelated signaling modulated the Dox resistance in prostate cancer cell DU-145 [15]. Cai et al. reported that IncRNA GBCDRlnc1 provoked chemoresistance through activation of autophagy in gallbladder cancer [16]. In myeloid leukemia cells, Yang et al. proposed that lncRNA linc00239 underlay the malignancies and chemoresistance against Dox by PI3K/Akt/mTOR signaling over-activation [17]. Knockdown assay performed by Dong et al. demonstrated that IncRNA HOXA-AS2 deficiency suppressed the chemoresistance through miR-520c-3p/S100A4 pathway [18]. Similar conclusion was drawn in colorectal cancer by Zhu et al. manifested that lncRNA XIST silencing greatly compromised the Dox resistance via increase of miR-124 and decrease of SGK1 [19]. Zhu et al. further disclosed that Fibronectin-1 regulated by lncRNA OIP5AS1/miR-200b-3p underlined the Dox resistance in osteosarcoma cells [20]. Zhou et al. suggested that Dox resistance in osteosarcoma provoked by lncRNA SNHG12 might be attributed to miR-320a/MCL1 signaling axis [21]. Here we for the first time provided evidence in support of the novel involvements of LINC00426 in the drug resistance in osteosarcoma, which predominantly mediated by negative regulation of miR-4319.

During the incidence of Dox resistance, number of microRNAs were identified to play critical roles in variety of human cancers. Liu et al. recently demonstrated the imposed Dox resistance in gastric cancer by exosomal transfer of miR-501 to specifically target BLID. MiR-134 was characterized to regulate resistance to Dox in human breast cancer cells via down-regulation of ABCC1 [22], while miR-132 and miR-212 were identified by Xie et al. to mediated Dox resistance through suppression of PTEN-AKT/NF-kB pathway [23]. In hepatocellular carcinoma, Tian et al. suggested that miR-760 inhibited Dox resistance via targeting Notch1/Hes1-PTEN/Akt signaling [24]. In osteosarcoma, several miRs were proposed to link with the occurrence of Dox resistance. Lin et al. firstly demonstrated that miR-184/BCL2L1 signaling axis contributed to Dox resistance in osteosarcoma [25]. Zhou et al. subsequently suggested that lncRNA SNHG12 modulated Dox resistance through miR-320a/MCL1 signaling [21]. The study performed by Zhang et al. indicated that miR-301a regulated Dox resistance via modulating AMPactivated protein kinase alpha 1 [26]. More recently, an intriguing investigation conducted by Wang et al. showed that IncRNA CTA significantly sensitized osteosarcoma cells to Dox treatment via inhibition of autophagy [27]. Noting worthily, our data highlighted the critical role of miR-4319 in the Dox resistance in osteosarcoma, which might hold great therapeutic promises for the future exploitations.

\section{Conclusion}

In summary, we uncovered the mechanistic contribution of LINC00426/miR-4319 to Dox resistance in osteosarcoma, which warranted further investigation and translational developments. Our study has, for the first time, unraveled the important clinical roles of LINC00426 in osteosarcoma response to Dox and identified its competing miR, which has shed new light on the incidence of Dox resistance in this disease and offered new opportunity for therapeutic intervention.

\section{Abbreviations}

Dox: Doxorubicin; OS: Osteosarcoma; PCR: Polymerase chain reaction; CCK8: Cell counting kit-8; CT: Computed tomography; PET: Positron emission 
computed tomography; MRI: Magnetic resonance imaging; IncRNA: Long non-coding RNA; miRs: MicroRNAs; ATCC: American Type Culture Collection; FBS: Fetal bovine serum; PFA: Paraformaldehyde; PBS: Phosphate buffer saline

\section{Acknowledgments}

None.

\section{Authors' contributions}

Lulin Wang, Yi Luo, Yiquan Zheng, Lifeng Zheng and Wenxiang Lin conceived and designed the experiments; Wenxiang Lin and Zonglin Chen performed the experiments; Shichun Wu analysed and interpreted the data and contributed reagents, materials, analysis tools or data; Jinhong Chen and Yun Xie wrote the paper. The author(s) read and approved the final manuscript.

\section{Funding}

None.

\section{Availability of data and materials}

All data generated or analyzed during this study are included in this published article.

\section{Ethics approval and consent to participate}

The protocol was approved by the ethics commitment of Zhangzhou

Affiliated Hospital of Fujian Medical University.

\section{Consent for publication}

All authors approved this publication.

\section{Competing interests}

The authors declare that they have no competing interests.

Received: 27 February 2020 Accepted: 26 June 2020

Published online: 03 July 2020

\section{References}

1. Rickel K, Fang F, Tao J. Molecular genetics of osteosarcoma. Bone. 2017;102: 69-79.

2. Federman N, Bernthal N, Eilber FC, Tap WD. The multidisciplinary management of osteosarcoma. Curr Treat Options Oncol. 2009:10:82-93.

3. Makielski KM, Mills LJ, Sarver AL, Henson MS, Spector LG, Naik S, et al. Risk factors for development of canine and human osteosarcoma: a comparative review. Vet Sci. 2019:6:48

4. Li S, Sun W, Wang H, Zuo D, Hua Y, Cai Z. Research progress on the multidrug resistance mechanisms of osteosarcoma chemotherapy and reversal. Tumour Biol. 2015;36:1329-38.

5. Ransohoff JD, Wei Y, Khavari PA. The functions and unique features of long intergenic non-coding RNA. Nat Rev Mol Cell Biol. 2018;19:143-57.

6. Bartholdi D, Stray-Pedersen A, Azzarello-Burri S, Kibaek M, Kirchhoff M, Oneda B, et al. A newly recognized 13q12.3 microdeletion syndrome characterized by intellectual disability, microcephaly, and eczema/atopic dermatitis encompassing the HMGB1 and KATNAL1 genes. Am J Med Genet A. 2014;164A:1277-83.

7. Kapranov P, Cheng J, Dike S, Nix DA, Duttagupta R, Willingham AT, et al. RNA maps reveal new RNA classes and a possible function for pervasive transcription. Science. 2007:316:1484-8.

8. Guo LL, Song CH, Wang P, Dai LP, Zhang JY, Wang KJ. Competing endogenous RNA networks and gastric cancer. World J Gastroenterol. 2015; 21:11680-7.

9. Yang Y, Li H, Liu Y, Chi C, Ni J, Lin X. MiR-4319 hinders YAP expression to restrain non-small cell lung cancer growth through regulation of LIN28mediated RFX5 stability. Biomed Pharmacother. 2019;115:108956.

10. Bian S. miR-4319 inhibited the development of thyroid cancer by modulating FUS-stabilized SMURF1. J Cell Biochem. 2020;121(1):174-82.

11. Huang L, Zhang Y, Li Z, Zhao X, Xi Z, Chen H, et al. MiR-4319 suppresses colorectal cancer progression by targeting ABTB1. United European Gastroenterol J. 2019:7:517-28.

12. Trougakos IP, So A, Jansen B, Gleave ME, Gonos ES. Silencing expression of the clusterin/apolipoprotein $J$ gene in human cancer cells using small interfering RNA induces spontaneous apoptosis, reduced growth ability, and cell sensitization to genotoxic and oxidative stress. Cancer Res. 2004;64: 1834-42.

13. Huarte M. The emerging role of IncRNAs in cancer. Nat Med. 2015;21:125361.

14. Nikolaou M, Pavlopoulou A, Georgakilas AG, Kyrodimos E. The challenge of drug resistance in cancer treatment: a current overview. Clin Exp Metastasis. 2018:35:309-18

15. Bai T, Liu Y, Li B. LnCRNA LOXL1-AS1/miR-let-7a-5p/EGFR-related pathway regulates the doxorubicin resistance of prostate cancer DU-145 cells. IUBMB Life. 2019;71:1537-51.

16. Cai Q, Wang S, Jin L, Weng M, Zhou D, Wang J, et al. Long non-coding RNA GBCDRlnc1 induces chemoresistance of gallbladder cancer cells by activating autophagy. Mol Cancer. 2019;18:82.

17. Yang Y, Dai W, Sun Y, Zhao Z. Long noncoding RNA linc00239 promotes malignant behaviors and chemoresistance against doxorubicin partially via activation of the PI3K/Akt/mTOR pathway in acute myeloid leukaemia cells. Oncol Rep. 2019;41:2311-20.

18. Dong X, Fang Z, Yu M, Zhang L, Xiao R, Li X, et al. Knockdown of Long noncoding RNA HOXA-AS2 suppresses Chemoresistance of acute myeloid leukemia via the miR-520c-3p/S100A4 Axis. Cell Physiol Biochem. 2018;51: 886-96.

19. Zhu J, Zhang R, Yang D, Li J, Yan X, Jin K, et al. Knockdown of Long noncoding RNA XIST inhibited doxorubicin resistance in colorectal cancer by upregulation of miR-124 and downregulation of SGK1. Cell Physiol Biochem. 2018;51:113-28.

20. Kun-Peng Z, Chun-Lin Z, Xiao-Long M, Lei Z. Fibronectin-1 modulated by the long noncoding RNA OIP5-AS1/miR-200b-3p axis contributes to doxorubicin resistance of osteosarcoma cells. J Cell Physiol. 2019;234:692739.

21. Zhou B, Li L, Li Y, Sun H, Zeng C. Long noncoding RNA SNHG12 mediates doxorubicin resistance of osteosarcoma via miR-320a/MCL1 axis. Biomed Pharmacother. 2018;106:850-7.

22. Liu X, Lu Y, Xu Y, Hou S, Huang J, Wang B, et al. Exosomal transfer of miR501 confers doxorubicin resistance and tumorigenesis via targeting of BLID in gastric cancer. Cancer Lett. 2019:459:122-34.

23. Xie M, Fu Z, Cao J, Liu Y, Wu J, Li Q, et al. MicroRNA-132 and microRNA-212 mediate doxorubicin resistance by down-regulating the PTEN-AKT/NFkappaB signaling pathway in breast cancer. Biomed Pharmacother. 2018; 102:286-94.

24. Tian T, Fu X, Lu J, Ruan Z, Nan K, Yao Y, et al. MicroRNA-760 inhibits doxorubicin resistance in hepatocellular carcinoma through regulating Notch1/Hes1-PTEN/Akt signaling pathway. J Biochem Mol Toxicol. 2018;32 e22167.

25. Lin BC, Huang D, Yu CQ, Mou Y, Liu YH, Zhang DW, et al. MicroRNA-184 modulates doxorubicin resistance in osteosarcoma cells by targeting BCL2L1. Med Sci Monit. 2016;22:1761-5.

26. Zhang Y, Duan G, Feng S. MicroRNA-301a modulates doxorubicin resistance in osteosarcoma cells by targeting AMP-activated protein kinase alpha 1. Biochem Biophys Res Commun. 2015;459:367-73.

27. Wang Z, Liu Z, Wu S. Long non-coding RNA CTA sensitizes osteosarcoma cells to doxorubicin through inhibition of autophagy. Oncotarget. 2017;8: $31465-77$

\section{Publisher's Note}

Springer Nature remains neutral with regard to jurisdictional claims in published maps and institutional affiliations.

Ready to submit your research? Choose BMC and benefit from:

- fast, convenient online submission

- thorough peer review by experienced researchers in your field

- rapid publication on acceptance

- support for research data, including large and complex data types

- gold Open Access which fosters wider collaboration and increased citations

- maximum visibility for your research: over $100 \mathrm{M}$ website views per year

At BMC, research is always in progress.

Learn more biomedcentral.com/submissions 